\title{
FOLFIRI Combined with Bevacizumab as First-Line Treatment for Metastatic Colorectal Cancer Patients with Hyperbilirubinemia after UGT1A1 Genotyping
}

\author{
Yung-Sung Yeh ${ }^{a, c}, g$ Meng-Lin Huang ${ }^{b}$ Se-Fen Chang ${ }^{d}$ Chin-Fan Chen ${ }^{a, c}$ \\ Huang-Ming $\mathrm{Hu}^{\mathrm{e}}$ Jaw-Yuan Wang ${ }^{\mathrm{c}, \mathrm{f}, \mathrm{g}}$ \\ ${ }^{a}$ Department of Emergency Medicine, Kaohsiung Municipal Hsiao-Kang Hospital, ${ }^{b}$ Department of Surgery, ZuoYing \\ Armed Forces General Hospital, Departments of ${ }^{\mathrm{C}}$ Surgery, ${ }^{\mathrm{d}}$ Nursing and ${ }^{\mathrm{e}}$ Internal Medicine and ${ }^{\mathrm{f}}$ Cancer Center, \\ Kaohsiung Medical University Hospital, and ${ }^{9}$ Department of Surgery, Faculty of Medicine and Graduate Institute of \\ Clinical Medicine, College of Medicine, Kaohsiung Medical University, Kaohsiung, Taiwan, ROC
}

\section{Key Words}

UGT1A1 · Liver dysfunction · Hyperbilirubinemia · FOLFIRI · Bevacizumab

\begin{abstract}
Objective: To report a metastatic colorectal cancer patient with hyperbilirubinemia treated with a combination of bevacizumab and FOLFIRI (5-fluorouracil, leucovorin, and irinotecan) using uridine diphosphate glucuronosyl transferase (UGT1A1) genotyping. Clinical Presentation and Intervention: A 46-year-old male was diagnosed with rectosigmoid colon cancer with liver metastases and hyperbilirubinemia presenting with severe jaundice. UGT1A1 genotyping was used before therapy to ascertain whether genotype-adjusted dosages of irinotecan plus bevacizumab could alleviate the toxicity. Then, the patient was treated with FOLFIRI. Conclusion: The FOLFIRI regimen was successfully used in this patient without concerns regarding toxicity.

(c) 2014 S. Karger AG, Basel
\end{abstract}

\section{Introduction}

The current standard first-line treatment for metastatic colorectal cancer (mCRC) includes FOLFOX (oxaliplatin plus infusional 5-fluorouracil and leucovorin) or FOLFIRI (5-fluorouracil, leucovorin, and irinotecan) plus biological agents. Irinotecan is a camptothecin derivative that exerts cytotoxic effects on cancer cells by inhibiting topoisomerase I, and FOLFIRI has been proven to be effective for the first-line treatment of patients with $\mathrm{mCRC}[1,2]$. However, to date, there is no standard protocol for mCRC patients with bilirubin $>2 \mathrm{mg} / \mathrm{dl}$. Relevant studies of the clinical benefits of chemotherapy for patients with severe hyperbilirubinemia and jaundice are lacking [3].

$U G T 1 A 1^{*} 28$ is considered the main predictor of toxicity in irinotecan-treated mCRC. UGT1A1 genotyping, established as a predictive marker for irinotecan-induced severe neutropenia/diarrhea, has been approved by the Food and Drug Administration of the USA [4]. However, there is no predictive marker for irinotecan-based che-

\begin{tabular}{ll}
\hline KARGER & $\begin{array}{l}\text { ( ) 2014 S. Karger AG, Basel } \\
1011-7571 / 14 / 0235-0478 \$ 39.50 / 0 \quad \text { Karger }\end{array}$ \\
$\begin{array}{l}\text { E-Mail karger@karger.com } \\
\text { www.karger.com/mpp }\end{array}$ & $\begin{array}{l}\text { Thisis an Open Access article licensed under the terms of the } \\
\text { Creative Commons Attribution-NonCommercial 3.0 Un- } \\
\text { ported license (CC BY-NC) (www.karger.com/OA-license), } \\
\text { applicable to the online version of the article only. Distribu- } \\
\text { tion permitted for non-commercial purposes only. }\end{array}$
\end{tabular}

Prof. Jaw-Yuan Wang, MD, PhD

Division of Gastrointestinal and General Surgery, Department of Surgery

Kaohsiung Medical University Hospital, Kaohsiung Medical University

No. 100, Tzyou 1st Road, Kaohsiung 807, Taiwan (ROC)

E-Mail cy614112@ms14.hinet.net 

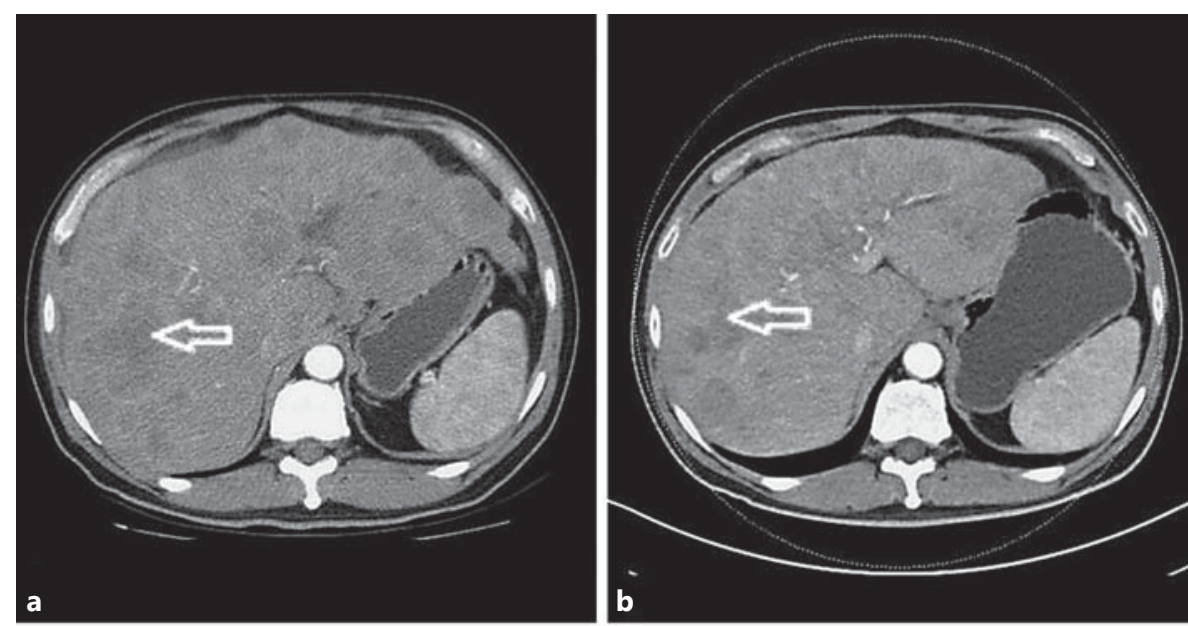

Fig. 1. a Image before chemotherapy, with more than 20 hepatic metastases ( $>70 \%$ liver replacement). b Image after 4 cycles of chemotherapy. Stable disease was noted on the CT scan. c A huge colon tumor can be seen over the rectosigmoid colon portion. d Shrinkage of the tumor was noted on the CT scan. The arrows indicate hepatic metastases in $\mathbf{a}+\mathbf{b}$; colon tumor in $\mathbf{c}+\mathbf{d}$.
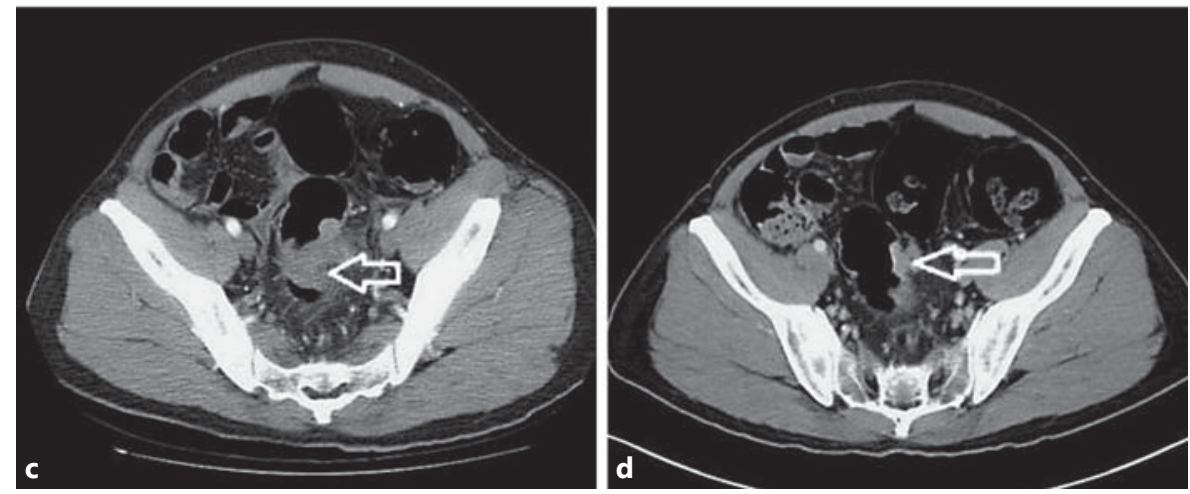

motherapy for the treatment of mCRC with severe liver dysfunction and prolonged hyperbilirubinemia. Hence, we report a case of severe hyperbilirubinemia with extensive liver metastasis from mCRC with FOLFIRI plus bevacizumab as first-line treatment.

\section{Case Report}

A 46-year-old male was referred to our hospital and was hospitalized with an initial presentation of a 3-month history of constipation, abdominal distension, poor appetite, body weight loss, and jaundice. Colonoscopy revealed a nearly $4 / 5$ circumferential obstructing ulcerative tumor in the rectosigmoid colon at approximately $12 \mathrm{~cm}$ from the anal verge, and pathological examination of a colonoscopic biopsy showed adenocarcinoma. No specific family history or past history was identified. Physical findings revealed icteric sclera and palpable hepatomegaly from the epigastrium to the right upper abdomen. Computed tomography (CT) of the abdomen and pelvis showed a huge rectosigmoid colon tumor approximately $6 \mathrm{~cm}$ in maximal diameter with more than 20 hepatic metastases ( $>70 \%$ liver replacement). The patient was finally diagnosed with rectosigmoid colon cancer with multiple liv- er metastases, UICC stage IV, based on the above imaging study. Abnormal laboratory findings included a total bilirubin level of $5.94 \mathrm{mg} / \mathrm{dl}$, an alanine aminotransferase level of $276 \mathrm{IU} / \mathrm{l}$, an aspartate aminotransferase level of $125 \mathrm{IU} / \mathrm{l}$, and a carcinoembryonic antigen level of 12,587 ng/ml. Genomic DNA, extracted from the peripheral blood, was subjected to PCR sequencing for genotyping of the promoter region of the UGT1A1 gene. The $(\mathrm{TA})_{\mathrm{n}}$ repeat of this gene showed 6/6 repeats. Due to poor liver function, jaundice, and the results of genotyping, the recommended dose of irinotecan $\left(180 \mathrm{mg} / \mathrm{m}^{2}\right)$ was underdosed in this mCRC patient with UGT1A1 $6 / 6$ or UGT1A1 6/7 gene polymorphism. The patient was treated with a regimen of FOLFIRI at an initial dosage of $120 \mathrm{mg} / \mathrm{m}^{2}$ administered over $2 \mathrm{~h}$ on day 1 followed by 5 -fluorouracil $(400 \mathrm{mg} /$ $\mathrm{m}^{2}$ as an i.v. bolus infusion followed by $2,400 \mathrm{mg} / \mathrm{m}^{2}$ i.v. infusion over a 46 -hour period plus bevacizumab $5 \mathrm{mg} / \mathrm{kg}$, repeated every 2 weeks). After 4 cycles of therapy, with the irinotecan dose being escalated from 120 to $180 \mathrm{mg} / \mathrm{m}^{2}$, the bilirubin level of the patient significantly decreased to $0.78 \mathrm{mg} / \mathrm{dl}$, the alanine aminotransferase level decreased to $27 \mathrm{IU} / \mathrm{l}$, the aspartate aminotransferase level decreased to $18 \mathrm{IU} / \mathrm{l}$, and the carcinoembryonic antigen level decreased to $2,165 \mathrm{ng} / \mathrm{ml}$. No chemotherapy-related grade 3 or above toxicities were noted in the first 4 cycles of treatment. Chemotherapy was well tolerated, with excellent performance. Normalization of all liver enzymes and liver metastases was confirmed by a CT scan and the disease was considered to be stabilized (fig. 1). Colo- 
Fig. 2. a Colonoscopy revealed a nearly $4 / 5$ circumferential obstructing ulcerative tumor. b The primary tumor prominently shrank. The arrows indicate primary colon tumor.
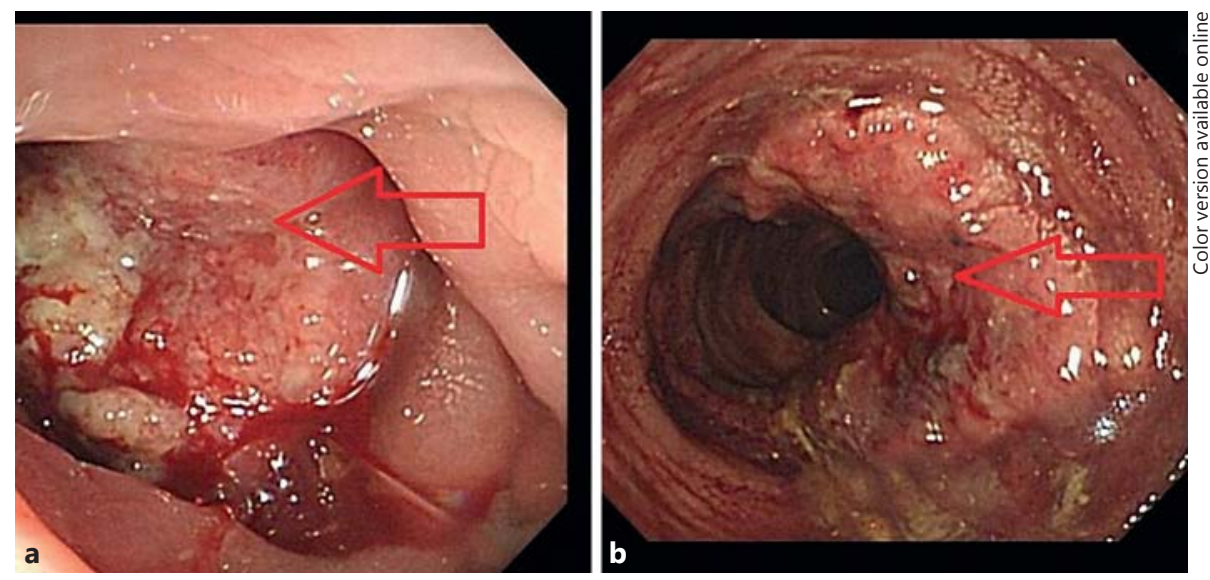

noscopy showed that the primary tumor had prominently shrunk (fig. 2). The patient received 12 cycles of FOLFIRI plus bevacizumab with clinical benefit. He survived 9 months from the time of first-line treatment with FOLFIRI combined with bevacizumab.

\section{Discussion}

This patient with rectosigmoid cancer and prolonged grade 4 hyperbilirubinemia was successfully treated with a regimen of FOLFIRI plus bevacizumab. After 4 cycles of this treatment, the therapy was well tolerated, with normalization of liver functions and predominant tumor shrinkage. We believe that, after excluding the possibility of obstructive jaundice, the use of novel genomic DNA analysis of the blood as in our current study can result in more favorable outcomes for such patients. mCRC patients with severe hyperbilirubinemia and liver dysfunction in which pharmacokinetic clearance is impaired often have a limited performance status and are at increased risk for chemotherapy-related complications $[5,6]$. In mCRC with liver metastasis and hyperbilirubinemia, there could be tumor burden-related (nonobstructive) or obstructive jaundice, and we should differentiate between them using laboratory examinations and imaging studies. The severe hyperbilirubinemia noted in these patients is more reflective of the underlying pathogenesis. Bilirubin data are currently used to exclude certain patients from receiving therapy (i.e. those with a serum bilirubin concentration of $>2 \mathrm{mg} / \mathrm{dl}$ should not be treated with irinotecan) $[5,7]$.

Irinotecan is converted into 7-ethyl-10-hydroxycamptothecin (SN-38) by a carboxylesterase and finally metabolized through the uridine diphosphate glucuro- nosyl transferase enzyme (UGT), predominantly by the UGT1A1 isoenzyme. Recently, Marcuello et al. [8] conducted a genotype-directed dose-finding study of irinotecan in combination with fluorouracil/leucovorin as first-line treatment for advanced CRC. Patients with homozygous polymorphism of the UGT1A1 promoter $\left(U G T 1 A 1^{*} 28\right)$ were more frequently associated with severe toxicity following irinotecan treatment. The genotype of the promoter polymorphism of the UGT1A1 gene in our patient was either UGT1A1 6/6 or UGT1A1 6/7. Individuals who are homozygous for the UGT1A1*28 allele are at increased risk for toxicity following the initiation of FOLFIRI treatment, and a reduced initial dose should be considered for patients known to be homozygous for the UGT1A $1^{*} 28$ allele (7 repeats). Heterozygous patients (carriers of one variant allele and one wild-type allele which results in intermediate UGT1A1 activity) may be at increased risk for toxicity; however, clinical results have been variable and such patients have been shown to tolerate normal starting doses [9]. Furthermore, the possible role of bevacizumab as a protective agent against the toxicity of irinotecan in these patients might need to be investigated. The addition of bevacizumab has been previously reported to improve the pathological response and protect against hepatic injury in patients treated with oxaliplatin-based chemotherapy for colorectal liver metastases [10].

\section{Conclusion}

The FOLFIRI regimen was successfully used in our patient with liver function abnormalities caused by mCRC without concerns regarding toxicity. 


\section{Acknowledgements}

The authors acknowledge the contribution to data collection made by the Colorectal Cancer Group of the Cancer Center of Kaohsiung Medical University Hospital. This study was supported by grants from the Excellence for Cancer Research Center Grant through funding from the Ministry of Health and Welfare, Executive Yuan, Taiwan, ROC (MOHW103-TD-B-111-05), and the Grant of Biosignature in Colorectal Cancers, Academia Sinica, Taiwan, ROC.

\section{References}

-1 Ishida H, Fujita K, Akiyama Y, et al: Regimen selection for first-line FOLFIRI and FOLFOX based on UGT1A1 genotype and physical background is feasible in Japanese patients with advanced colorectal cancer. Jpn J Clin Oncol 2011;41:617-623.

-2 Sanli UA, Karabulut B, Uslu R, et al: Singleagent irinotecan for recurrent/metastatic colorectal cancer: a retrospective analysis. Med Princ Pract 2006;15:288-292.

-3 Shimura T, Kataoka H, Hirata Y, et al: Metastatic colorectal cancer with severe liver dysfunction successfully treated using FOLFOX therapy. J Gastrointest Cancer 2011;42:68-72.

4 Cecchin E, Innocenti F, D'Andrea M, et al: Predictive role of the UGT1A1, UGT1A7, and UGT1A9 genetic variants and their haplotypes on the outcome of metastatic colorectal cancer patients treated with fluorouracil, leucovorin, and irinotecan. J Clin Oncol 2009;27: 2457-2465.
-5 Fakih MG: 5-Fluorouracil leucovorin and oxaliplatin (FOLFOX) in the treatment of metastatic colon cancer with severe liver dysfunction. Oncology 2004;67:222-224.

6 Venook AP, Enders Klein C, Fleming G, et al: A phase I and pharmacokinetic study of irinotecan in patients with hepatic or renal dysfunction or with prior pelvic radiation: CALGB 9863. Ann Oncol 2003;14:17831790.

7 Walia T, Quevedo JF, Hobday TJ, et al: Colorectal cancer patients with liver metastases and severe hyperbilirubinemia: a consecutive series that explores the benefits and risks of chemotherapy. Ther Clin Risk Manag 2008;4:1363-1366.
8 Marcuello E, Páez D, Paré L, et al: A genotypedirected phase I-IV dose-finding study of irinotecan in combination with fluorouracil/ leucovorin as first-line treatment in advanced colorectal cancer. Br J Cancer 2011;105:5357.

9 Fuchs CS, Marshall J, Mitchell E, et al: Randomized, controlled trial of irinotecan plus infusional, bolus, or oral fluoropyrimidines in first-line treatment of metastatic colorectal cancer: results from the BICC-C Study. J Clin Oncol 2007;25:4779-4786.

10 Ribero D, Wang H, Donadon M, et al: Bevacizumab improves pathologic response and protects against hepatic injury in patients treated with oxaliplatin-based chemotherapy for colorectal liver metastases. Cancer 2007; 110:2761-2767. 